\title{
Suporte nutricional no recém-nascido doente ou prematuro
}

\author{
Nutritional support in the ill or premature \\ newborn infant
}

\author{
Mário Cícero Falcão(1)
}

\begin{abstract}
Falcão MC. Suporte nutricional no recém-nascido doente ou prematuro. Rev Med (São Paulo) 2003 jan.dez.;82(1-4):11-21.

RESUMO: Ao nascimento, o recém-nascido tem que se adaptar a uma nova maneira de nutrição, passando de um afluxo constante de praticamente todos os nutrientes, para uma alimentação intermitente, tendo que utilizar suas funções digestórias. Recém-nascidos de termo normais rapidamente superam essas barreiras. No entanto, recém-nascidos pré-termo ou mesmo recém-nascidos de temo doentes normalmente apresentam condições adversas que dificultam a sua nutrição plena. Nestes casos, um suporte nutricional deve ser instituído para que se possa promover uma nutrição adequada. Este suporte normalmente consiste na avaliação nutricional, no cálculo das necessidades nutricionais e na monitorização da terapia nutricional individualizada.
\end{abstract}

DESCRITORES: Apoio nutricional. Avaliação nutricional. Recém-nascido. Prematuro. Necessidades nutricionais. Nutrição infantil.

O estado nutricional de uma criança ao nascer varia de acordo com as condições de vida intra-uterina as quais esteve submetida. A adequação nutricional do feto pode influenciar de forma significante a morbidade e a mortalidade do recém-nascido (RN). O objetivo principal do neonatologista é identificar esse $\mathrm{RN}$ com risco de apresentar complicações decorrentes da inapropriada taxa de crescimento intra-uterina, seja ela insuficiente ou exagerada e antecipar-se a essas complicações, prevenindo suas seqüelas.
O crescimento fetal é influenciado por diversos fatores, como os genéticos, hormonais, ambientais, placentários, saúde materna, incluindo sua própria condição nutricional e oferta de nutrientes ${ }^{1}$. De acordo com o grau e o tipo de influência exercida por alguns desses fatores, detecta-se benefícios ou prejuízos no crescimento intra-uterino ${ }^{1}$. É importante ressaltar que o padrão de crescimento fetal "normal", que alcança uma velocidade muito grande no terceiro trimestre da gestação, é bastante suscetível à influência dos fatores citados acima.

\footnotetext{
${ }^{(1)}$ Doutor em Pediatria pela Faculdade de Medicina da Universidade de São Paulo. Médico do Berçário Anexo à Maternidade do Hospital das Clínicas da Faculdade de Medicina da Universidade de São Paulo. Professor Colaborador da Disciplina de Pediatria Neonatal do Departamento de Pediatria da Faculdade de Medicina da Universidade de São Paulo.

Endereço para correspondência: R. Vieira de Moraes, 45 - Apto. 51. CEP: 04617-010 - Brooklin, São Paulo, SP. e-mail: falcao@node1.com.br
} 
A composição corpórea do feto modifica-se no decorrer da gestação, ocorrendo progressiva diminuição da água total e extracelular e dos íons sódio e cloro e concomitante aumento da água intracelular, dos íons cálcio e magnésio, de proteínas, gorduras e glicogênio ${ }^{2}$.

Na prática diária neonatal, as principais medidas antropométricas utilizadas são o peso, o comprimento e o perímetro cefálico (PC). As outras relações, como por exemplo, a prega adiposa tricipital, a circunferência braquial e o cálculo da massa muscular do braço devem ser reservadas para fins específicos.

Toda medida antropométrica só terá valor diagnóstico e prognóstico quando realizada corretamente, de preferência pelo mesmo observador e repetida a intervalos pré-determinados.

Recomenda-se a medida de peso uma a duas vezes por dia, o comprimento e o perímetro cefálico uma vez por semana. Estes dados devem ser transportados para gráficos antropométricos de idade gestacional (IG), onde serão feitas as devidas comparações quanto a sua "normalidade", ressaltando-se que é necessário o uso da idade pós-conceptual, que é a IG acrescida da idade cronológica.

As determinações laboratoriais, além de não poderem ser aplicadas em todas as situações, mostram uma avaliação estática e, só são úteis, quando utilizadas em conjunto com a antropometria e a avaliação dinâmica, ou seja, a monitorização da velocidade de crescimento no período neonatal ${ }^{4}$.

É de suma importância que se estabeleça um roteiro ordenado de avaliação nutricional do RN. Segue abaixo uma sugestão de roteiro para tal avaliação ${ }^{5}$ :

1) Anamnese - com ênfase nos dados maternos;

2) Exame físico - já corresponde a uma avaliação sumária da nutrição intra-uterina, da avaliação da massa muscular e da gordura subcutânea;

3) Classificação do RN - será detalhado adiante;

4) Antropometria - será detalhado adiante;

5) Avaliação da oferta nutricional, basicamente através de balanços;

6) Composição corpórea - através de métodos biofísicos (bio-impedância) e por imagem (ultra-sonografia e tomografia computadorizada), com padrões pouco estabelecidos, principalmente no recém-nascido pré-termo (RNPT);

7) Avaliação laboratorial - será detalhado adiante.

\section{Classificação nutricional do recém-nascido:} $(\mathrm{PN})^{5}$

1. Classificação do RN pelo peso de nascimento

- $\mathrm{PN}<2500 \mathrm{~g}{ }^{\circledR}$ RN de baixo peso (RNBP);

- $\mathrm{PN}<1500 \mathrm{~g}{ }^{\circledR} \mathrm{RN}$ de muito baixo peso (RNMBP);

- $\mathrm{PN}<1000 \mathrm{~g}{ }^{\circledR} \mathrm{RN}$ de muitíssimo baixo peso (RNMMBP);

- $\mathrm{PN}<800 \mathrm{~g}{ }^{\circledR}$ RN microprematuro.

2. Classificação do RN pela idade gestacional (IG):
- Critérios para a determinação da IG;

a) IG materna, segundo a regra de Naegele, que determina a gestação normal em 280 dias, de acordo com a data da última menstruação;

b) IG avaliada por método ultra-sonográfico, realizado até a $12^{\mathrm{a}}$ semana de gestação ${ }^{6}$;

c) cálculo pós-natal da IG, pela avaliação clínica, com dados antropométricos, exame físico e neurológico, aplicando-se então, os métodos de Capurro, Dubowitz, New Ballard, etc ${ }^{7,8,9}$.

Na determinação da IG, os critérios acima descritos devem ser utilizados em ordem crescente, de forma que, ante uma IG materna confiável, os itens b e c serão automaticamente desconsiderados.

- IG $<37$ sem. ${ }^{\circledR}$ RNPT.

- IG entre 37 e 42 sem. ${ }^{\circledR}$ RN de termo (RNT).

- IG > 42 sem ${ }^{\circledR}$ RN pós-termo.

3. Classificação do RN por PN e IG:

É realizada através das curvas de crescimento fetal, de acordo com as diferentes idades gestacionais, utilizando-se o critério de percentis ${ }^{3,10}$. 10 e 90.

- Adequado para a IG (AIG) ® entre os percentis

- Pequeno para a IG(PIG) ${ }^{\circledR}$ abaixo do percentil 10.

- Grande para a IG (GIG) ® acima do percentil 90.

4. Classificação do recém-nascido pela relação entre o peso de nascimento e o peso no percentil 50 da curva de crescimento fetal (para a mesma idade gestacional).

Inicialmente, esta relação, também chamada de "Critério de Kramer" "11 foi usada para mostrar o retardo de crescimento intra-uterino. Relações abaixo de 0,70 revelavam um importante agravo nutricional. Atualmente, também utilizamos esta classificação para a adequação nutricional do RN, a saber:

- $<0,85$ \& RN PIG;

- entre 0,90 e 1,10® RN AIG;

- >1,10® RNGIG.

\section{Antropometria}

Avalia as relações entre crescimento fetal intrauterino, estado nutricional e morbimortalidade perinatal, predizendo a evolução pós-natal.

Ao nascimento, a antropometria tem uma relação direta com a qualidade do crescimento fetal; posteriormente, em avaliações seriadas, mostra o crescimento pós-natal e avalia a terapia nutricional ${ }^{11}$.

\section{Peso}

O peso de nascimento varia com a idade gestacional, o estado nutricional e hidroeletrolítico materno e do RN, sendo considerado o "gold standard" para a avaliação do crescimento perinatal e sua alteração mostra distúrbios perinatais agudos e crônicos. A sua mensuração deve ser feita uma a duas vezes por dia e o RN com oferta nutricional 
adequada deve crescer de 20 a $40 \mathrm{~g}$ por dia, conforme as curvas de crescimento intra-uterino. Vale a pena lembrar que os RN apresentam uma perda de peso "fisiológica", que varia de 10 a $20 \%$, sendo inversamente proporcional à IG.

\section{Comprimento}

Esta medida reflete o potencial genético do crescimento e sofre menor influência ante uma nutrição fetal inadequada, além de não se alterar com o estado de hidratação ${ }^{11}$. Deve ser realizada ao nascimento e, posteriormente, uma vez por semana, esperando-se crescimento de $1 \mathrm{~cm}$ por semana, conforme a comparação com as curvas de crescimento intra-uterino.

\section{Perímetro cefálico}

A medida do PC, até os 6 meses de idade, tem relação direta com o tamanho do encéfalo e, o seu aumento proporcional indica crescimento adequado e melhor prognóstico neurológico ${ }^{11}$. É a medida antropométrica que é menos afetada por uma nutrição inadequada e é a primeira que cresce ao se atingir uma oferta protéico-calórica ideal. Espera-se crescimento de $1 \mathrm{~cm}$ por semana, conforme a comparação com as curvas de crescimento intra-uterino.

A primeira medida, normalmente realizada entre 6 a 12 horas de vida, necessita de confirmação, 48 a 72 horas após, devido à acomodação dos ossos do crânio.

4. Dobras cutâneas

A avaliação seriada dessa medida antropométrica promove uma estimativa da quantidade e da incorporação de gordura. No período neonatal utilizam-se as dobras bicipital, tricipital e escapular, salientando-se que a presença de edema pode alterar a aferição, porém, determinadas técnicas minimizam esses erros.

Atualmente, já existe padrão de comparação das dobras por IG, porém é um método mais laborioso, necessitando de três aferições e dependente de treino do observador $^{12}$.

\section{Perímetro braquial}

Esta aferição antropométrica avalia a massa muscular e a quantidade de gordura do braço. É uma técnica fácil de ser realizada, e tem relação direta e linear com o "status" nutricional do RN. Já existem curvas que relacionam o perímetro braquial com o peso e a IG, confirmando a relação diretamente proporcional à maturidade do RN. Em RNPT é uma medida mais acurada do que o peso e o comprimento, quando em avaliações seriadas $^{13,14,15}$.

\section{Relação perímetro braquial e cefálico}

Essa fração fornece informações sobre a proporcionalidade do crescimento e tem uma relação linear e direta com a IG. Ao nascimento ela representa a nutrição fetal e portanto, tem uma relação com a morbidade perinatal, principalmente frente ao retardo de crescimento intra- uterino. A sua avaliação seriada reflete o aporte calóricoprotéico neonatal ${ }^{13}$.

\section{7. Índice ponderal}

A melhor maneira para se avaliar a relação entre peso e comprimento é através do Índice Ponderal de Rohrer $(\mathrm{IP})^{3}$,

$\mathrm{IP}=\underline{\mathrm{PN}(\mathrm{g}) \times 100} \quad$ PN: peso ao nascer $\mathrm{CN}^{3}(\mathrm{~cm}) \quad \mathrm{CN}$ : comprimento ao nascer

cálculo este mais fidedigno para se demonstrar a desnutrição intra-uterina.

- IP entre os percentis 10 e 90:

- RNPIG simétrico ou proporcionado, ocorre na desnutrição materna crônica.

- IP menor que o percentil 10:

RNPIG assimétrico ou desproporcionado, ocorre na desnutrição intra-uterina aguda.

A importância desta classificação reflete-se na evolução destas crianças, pois os RNPIG proporcionados podem cursar com déficit pondo-estatural e do desenvolvimento neuropsicomotor.

\section{Avaliação laboratorial}

Basicamente avalia o "status" energético-protéico, podendo ser dividida $\mathrm{em}^{4}$ :

A) Avaliação do "status" das proteínas séricas de curta duração:

a) pré-albumina: esta determinação pode ser alterada pela presença de infecções ou traumas, doenças hepáticas ou renais e corticoterapia, sendo dependente de uma função hepática íntegra. Além disso, como ela se altera com a IG, ainda não existem padrões definidos para o RNPT;

b) proteínas ligadas ao retinol: também dependem da função hepática e das concentrações de zinco e de vitamina A. Além disso, o RNPT pode ter níveis menores dessa proteína pela deficiência de vitamina A e não por síntese diminuída;

c) transferrina: eleva-se com a IG e com o peso fetal, porém altera-se com a deficiência de ferro;

\section{B) Avaliação do "status" das proteínas séricas de} longa duração:

a) albumina: seus níveis podem estar normais, a despeito de uma nutrição inadequada e, para RNPT os valores ainda não estão totalmente estabelecidos, podendo estar mais baixa tanto por menor produção quanto por "turnover" mais acelerado. Esta proteína é um melhor marcador de uma avaliação nutricional mais prolongada, não servindo para avaliações em curtos períodos de tempo.

\section{nascido}

\section{Necessidades nutricionais basais do recém-}

A) Necessidades hídricas ${ }^{16,17}$ :

- Valores recomendados para o $1^{\circ}$ dia de vida

N T: 60 ml/kg/dia; 
RNPT: $80 \mathrm{ml} / \mathrm{kg} / \mathrm{dia}$.

- Valores recomendados para o final da $1^{\mathrm{a}}$ semana de vida:

$120 \mathrm{a} 160 \mathrm{ml} / \mathrm{kg} / \mathrm{dia}$.

B) Necessidades calórico-energéticas: deve-se atingir $120 \mathrm{kcal} / \mathrm{kg} /$ dia na $2^{\mathrm{a}}$ semana de vida, oferecendo-se calorias na proporção de $40 \%$ em hidratos de carbono e $60 \%$ em gorduras ${ }^{18,19}$.

Obs: deve-se respeitar a relação nitrogênio/calorias não protêicas de 1/160. $\operatorname{dia}^{18,19}$.

C) Necessidades lipídicas: em torno de 1 a 1,5 g/kg/

Obs: caso seja prescrito um valor maior, fazer controles de triglicérides e colesterol séricos.

D) Necessidades de eletrólitos, minerais e microelementos ${ }^{20}$ :

$\mathrm{Na}: 2$ a $3 \mathrm{mEq} / \mathrm{kg} / \mathrm{dia}$; até $8 \mathrm{mEq} / \mathrm{kg} /$ dia em RNMBP;

$\mathrm{K}: 2$ a $3 \mathrm{mEq} / \mathrm{kg} / \mathrm{dia}$;

Cl: 1,5 a $2,5 \mathrm{mEq} / \mathrm{kg} / \mathrm{dia}$;

Mg: no RNPT: $8 \mathrm{mg} / \mathrm{kg} / \mathrm{dia}$, no RNMBP: $10 \mathrm{mg} / \mathrm{kg} / \mathrm{dia}$;

Ca: no RNPT: $200 \mathrm{mg} / \mathrm{kg} / \mathrm{dia}$; no RNMBP: $240 \mathrm{mg} / \mathrm{kg} / \mathrm{dia}$;

P: no RNPT: $120 \mathrm{mg} / \mathrm{kg} / \mathrm{dia}$; no RNMBP: $140 \mathrm{mg} / \mathrm{kg} / \mathrm{dia}$;

Zn: 100 a 300 mg/kg/dia; no RNMBP: até $500 \mathrm{mg} / \mathrm{kg} / \mathrm{dia}$;

$\mathrm{Cu}: 20 \mathrm{mg} / \mathrm{kg} / \mathrm{dia}$, no RNMBP: até 90 mg/kg/dia;

Mn: $1 \mathrm{mg} / \mathrm{kg} / \mathrm{dia}$;

Cr: $0,2 \mathrm{mg} / \mathrm{kg} / \mathrm{dia}$;

Se: $2 \mathrm{mg} / \mathrm{kg} / \mathrm{dia}$;

Md: $0,25 \mathrm{mg} / \mathrm{kg} / \mathrm{dia}$.

E) Necessidades vitamínicas ${ }^{21}$ :

Vitamina A: 1500 a 2800 UI/dia;

Vitamina D: $800 \mathrm{UI} /$ dia;

Vitamina K: 0,5 a $1 \mathrm{mg}$ ao nascimento.

\section{Terapia nutricional no recém-nascido pré-termo}

A nutrição adequada do RNPT tem por objetivos suprir suas necessidades e promover crescimento e desenvolvimento adequados, sem efeitos indesejáveis (acidose metabólica, persistência do canal arterial, enterocolite necrosante, hipercolesterolemia, hiperuremia, hiperamonemia, entre outros $)^{22}$.

Recomendação de oferta diária para o $R N P T^{23}$

- Água $(\mathrm{ml} / \mathrm{kg}) \quad 80-160$

- Energia (Kcal/kg) 120-160 (média de 130)

- Proteínas (g/kg) 2,5-3,0

- Lípides $(\mathrm{g} / \mathrm{kg}) \quad 0,5-1,5$
- $\mathrm{Na}(\mathrm{mEq} / \mathrm{kg}) \quad 2,5-8,0$

- $\mathrm{K}(\mathrm{mEq} / \mathrm{kg}) \quad 2,0-3,0$

- $\mathrm{Ca}(\mathrm{mg} / \mathrm{kg}) \quad 200-240$

- $\mathrm{P}(\mathrm{mg} / \mathrm{kg}) \quad 100-140$

- $\mathrm{Mg}(\mathrm{mg} / \mathrm{kg}) \quad 6-12$

- $\mathrm{Cl}(\mathrm{mEq} / \mathrm{kg}) \quad 1,5-2,5$

Recomendação de vitaminas para o RNPT $T^{23}$

- D(UI) 400-800/dia

- A(UI) 1500-2500/dia

- E(UI) 6-12/dia

- K(mg) 1-2/semana

\section{Nutrição enteral}

O leite produzido pela própria mãe do RNPT é o mais indicado para a sua alimentação, porém, para os RN muito imaturos, este leite pode apresentar deficiências em relação a proteínas, energia, sódio, cálcio, fósforo, microelementos e algumas vitaminas, sendo então necessário o uso de aditivos do leite materno ${ }^{24,25}$.

Na impossibilidade do uso do leite materno, lançase mão de fórmulas especiais para prematuros que, apesar de não serem substitutas, preenchem algumas deficiências destas crianças, principalmente em relação a proteínas, cálcio e fósforo ${ }^{26}$.

O início da alimentação deve ser o mais precoce possível, assim que as condições clínicas o permitam. $\mathrm{O}$ volume, assim como o incremento deste, está intimamente relacionado ao peso do RN. O intervalo entre as mamadas pode variar entre 2 a 3 horas.

1. Sugestão de esquema alimentar para o RNPT

Peso de

nascimento: > $1250 \mathrm{~g}$ entre $1000-1250 \mathrm{~g}>1250 \mathrm{~g}$

Início (idade): $6 \mathrm{~h} \quad 24 \mathrm{~h} \quad 48 \mathrm{~h}$

Volume da $\quad 5 \mathrm{ml} \quad 2 \mathrm{ml} \quad 1 \mathrm{ml}$

Periodicidade: $3 / 3 \mathrm{~h} \quad 3 / 3 \mathrm{~h} \quad 2 / 2$ ou $3 / 3 \mathrm{~h}$

Incremento: $\quad 20 \mathrm{ml} / \mathrm{kg} / \mathrm{dia}$ (para qualquer peso)

\section{Rotina alimentar}

Normalmente utilizam-se as sondas nasogástricas e/ou orogástricas. As primeiras apresentam como vantagens uma fixação mais fácil e duradoura, porém, provocam um aumento na resistência das vias aéreas, inclusive, propiciando a ocorrência de apnéias.

Em relação ao uso de sondas intragástricas ou tranpilóricas, estudos não demonstraram redução de incidência de pneumonias aspirativas nem melhor tolerância ao volume alimentar com a sonda jejunal.

Teoricamente, o uso da gavagem contínua reduziria a ocorrência de apnéias, de distensão abdominal e de refluxo gastro-esofágico, porém aumentaria a ocorrência de infecções (maior exposição dos tubos ao ambiente) e de alterações físico-químicas da dieta infundida. 
A gavagem gástrica contínua pode ser uma opção valiosa em RN imaturos, principalmente sob ventilação mecânica, pois, a oferta de uma quantidade pequena porém contínua de alimento no trato gastrointestinal propicia a nutrição trófica da mucosa, impedindo a translocação bacteriana e preparando o intestino para receber dieta láctea adequada posteriormente.

A sonda nasogástrica deve ser trocada a cada 24 horas e a orogástrica a cada 72 horas. Preconiza-se a administração da dieta através da gravidade e não em "bolo". Antes da cada oferta deve-se aspirar a sonda para se verificar a presença de resíduo e após a administração, o RN deverá ser colocado em decúbito ventral elevado.

Se o RNPT recusar a alimentação adequada ou apresentar resíduo gástrico deve-se detectar a causa. Caso ocorram vômitos, cianose ou distensão abdominal a dieta deve ser suspensa, pois, qualquer alteração quanto à aceitação alimentar pode ser o primeiro sinal de doença nessas crianças.

Em RN abaixo de 34 semanas de IG a alimentação deve sempre ser iniciada através de gavagem, contínua ou intermitente, orogástrica ou naso-jejunal. Entre 34 e 36 semanas, deve-se observar se o RN apresenta sucçãodeglutição coordenadas, para então, ser liberada a alimentação por via oral. Nos RNPT com 36 semanas, normalmente, o seio materno já pode ser prescrito desde as primeiras mamadas.

\section{Aditivos do leite humano}

O leite humano, além das vantagens nutricionais, proporciona inúmeras outras vantagens para o recémnascido, como defesas contra agentes infecciosos, promoção do trofismo gastrintestinal, melhor contato entre a mãe e o RN. No entanto, seu conteúdo mineral e protéico, em situações específicas, pode não atender adequadamente as necessidades nutricionais do RNPT ${ }^{27}$.

As recomendações para a oferta de cálcio e fósforo não são unânimes; a Academia Americana de Pediatria sugere uma oferta de 200 a $250 \mathrm{mg} / \mathrm{kg} /$ dia de cálcio e de $120 \mathrm{mg} / \mathrm{kg} / \mathrm{dia}$ de fósforo, com o objetivo de obter uma incorporação mineral semelhante à intra-uterina. No entanto, a Sociedade Européia de Gastroenterologia e Nutrição Pediátrica recomenda uma oferta menor, em torno de $120 \mathrm{mg} / \mathrm{kg} / \mathrm{dia}$ de cálcio e de $70 \mathrm{mg} / \mathrm{kg} / \mathrm{dia}$ de fósforo para atender às necessidades nutricionais deste grupo de recém-nascidos, que seria suficiente para a incorporação adequada destes minerais ${ }^{28}$.

Para atender às necessidades nutricionais dos RNPT e para substituição do leite humano em situações em que as mães não conseguem oferecer seu próprio leite, fórmulas lácteas específicas foram industrializadas. No entanto, elas não conseguem substituir o leite humano completamente, pois não contêm substâncias que conferem proteção contra infecções, fatores de amadurecimento e trofismo intestinal, entre outras ${ }^{29,30}$.
Com o objetivo de utilizar as vantagens que o leite humano proporciona e torná-lo adequado para atender as necessidades nutricionais do RNPT têm sido realizados esforços para se obter soluções que adicionadas ao leite humano, atendam estas necessidades. Estes compostos, atualmente já disponíveis no mercado, são denominados aditivos do leite humano.

\section{Indicações e utilização}

Não existe uma recomendação unânime quanto à utilização destes aditivos, que podem ser sob a forma de pó ou líquido. No entanto, a maior parte dos estudos sugere o seu uso em RN com peso de nascimento menor que 1500 gramas, e outros, em RN com idade gestacional menor que 32 semanas. Existem sugestões para sua utilização em populações de risco, como por exemplo, em prematuros com doença pulmonar crônica, após cirurgias ou durante o uso de corticoterapia, ou seja, em situações que aumentem o catabolismo ou alterem a incorporação protéica e mineral ${ }^{28}$.

Atualmente, existem vários preparados para este fim, cujas composições variam em relação à quantidade e fonte protéica, cálcio, fósforo, presença e quantidade de oligoelementos e vitaminas, fonte de carboidratos (lactose ou polímeros de glicose, como a dextrino-maltose), que por sua vez influenciará a osmolaridade.

Recomenda-se iniciar a adição destes preparados quando o RN estiver recebendo pelo menos $100 \mathrm{ml} / \mathrm{kg} / \mathrm{dia}$ de leite humano ou 50 a $80 \%$ das suas necessidades nutricionais por via enteral. Sugere-se que esta introdução seja gradual, conforme o esquema abaixo:

- início - 1/4 da dose recomendada;

- após 2 a 3 dias, aumenta-se para 1/2 da dose recomendada;

- após 2 a 3 dias aumenta-se para 2/3 da dose recomendada e;

- após 2 a 3 dias oferece-se a quantidade total recomendada.

\section{Nutrição enteral mínima}

O jejum prolongado, por seus efeitos deletérios no epitélio intestinal (atrofia da mucosa, diminuição da absorção e ausência de competição da flora intestinal), propicia a translocação bacteriana, aumentando a chance de ocorrer enterocolite necrosante e sepse. Este efeito pode ser minimizado pela oferta enteral precoce, pois a fermentação bacteriana dos carboidratos resulta em ácidos graxos de cadeia curta, principalmente o butírico, que apresentam uma função trófica sobre o epitélio intestinal. Além disso, a nutrição enteral melhora a tolerância à glicose, por gerar precursores neoglicogênicos (piruvatos, lactatos, alanina e glicerol), ácidos graxos não esterificados e corpos cetônicos (beta-hidroxibutirato e acetoacetatos) ${ }^{31}$.

Existe uma grande discussão a respeito da oferta enteral em RN infectados, pois a própria alimentação pode 
provocar a translocação bacteriana, a enterocolite e a sepse. Por outro lado, o jejum, levando à atrofia da mucosa e à alteração da flora, com predominância de "germes livres", também aumenta a ocorrência de infecções. Este estado de jejum também vai contra o processo de maturação natural do intestino, que começa na vida intra-uterina, onde o líquido amniótico deglutido tem papel nutricional sobre mucosa intestinal, propiciando a formação de uma barreira intestinal íntegra ${ }^{31,32,33}$.

A flora intestinal do RN, que normalmente começaria a ser colonizado por germes da flora vaginal materna, é alterada por alguns fatores: parto cesariano, anti-sepsia e uso de antibióticos da largo espectro. Estes fatores levariam ao rompimento do equilíbrio da barreira intestinal, com crescimento anormal da referida flora e facilitando a translocação bacteriana ${ }^{31}$.

A prática da nutrição enteral mínima, já nos primeiros dias de vida, apresenta alguns aspectos vantajosos para se prevenir uma infecção ou mesmo, para se evitar a progressão de um quadro infeccioso já instalado, além de não aumentar a incidência da enterocolite.

Esta nutrição trófica pode ser iniciada nos primeiros dias de vida, com 0,5 a $1 \mathrm{ml} / \mathrm{h}$ de água destilada, através de infusão orogástrica contínua. Este procedimento tem por finalidade repor a água livre, manter a motilidade intestinal e preparar o intestino para receber a dieta láctea. No momento em que o RN apresentar alguma estabilidade, devese iniciar a nutrição enteral hipocalórica com leite materno, através de infusão orogástrica contínua, na velocidade de 0,5 a $1 \mathrm{ml} /$ hora, aumentando-se paulatinamente até se atingir uma nutrição enteral adequada.

\section{Nutrição parenteral no recém-nascido}

$\mathrm{O}$ aporte nutricional adequado, de fundamental importância, especialmente para os RN de muito baixo peso, encontra dificuldades diversas no que concerne à nutrição enteral inicial. A menor tolerância presente nessas crianças, mesmo sem patologias relevantes, deve-se, em parte, ao menor comprimento do intestino, à menor motilidade intestinal, funções diminuídas de enzimas intestinais, imaturidade da regulação do fluxo sanguíneo intestinal e da imaturidade (com risco de enterocolite necrosante) $)^{31}$.

Assim, tem importância fundamental o recurso da nutrição parenteral (NP), que pode coexistir, por período variado, com a nutrição enteral mínima.

Como norma prática, o objetivo inicial da NP não é o ganho de peso, mas o fornecimento de calorias e nitrogênio suficientes para prevenir catabolismo e promover balanço nitrogenado positivo. Os RN submetidos à NP por períodos mais prolongados necessitam calorias adicionais para ganho ponderal.

\section{Indicações}

A NP é indicada quando a alimentação enteral não for possível ou quando as necessidades calóricas e nutricionais, fornecidas por via enteral, não forem suficientes. Normalmente indica-se a NP em RN com peso de nascimento inferior a $1500 \mathrm{~g}$, submetidos à ventilação mecânica, na presença de sepse, de asfixia perinatal (alto risco para desenvolver enterocolite necrosante), de patologias cirúrgicas e em pós-operatório, ou ante qualquer patologia que impeça a alimentação enteral ${ }^{34}$.

A recomendação atual é a introdução precoce, ou seja até 48 horas de vida, desde que não haja contraindicação da NP.

\section{Vias de administração}

A NP pode ser administrada através de veias periféricas ou cateter central. A NP periférica está indicada para os RN que vão utilizá-la por período relativamente curto (10-14 dias).

A concentração de glicose nas soluções de NP periférica deve limitar-se a $12,5 \%$ pelo risco de esclerose venosa e lesões cutâneas. A NP periférica em combinação com as soluções de lipídes podem ofertar $60-80 \mathrm{kcal} / \mathrm{kg} /$ dia permitindo balanço nitrogenado positivo.

A NP através de cateter central está mais indicada para os RN com falta de acesso venoso, aumento das necessidades calóricas ou que necessitem restrição hídrica e especialmente, quando utilizada por período superior a 2 semanas. Dá-se preferência aos cateteres de silicone introduzido por via percutânea; na falta destes as dissecções venosas, com técnica de tunelização, estão indicadas. A ponta do cateter deve localizar-se na junção da veia cava com o átrio direito. Os cateteres umbilicais tem maior risco de infecção, devendo ser evitados. A via central também é a preferencial nos RN que recebem alta hospitalar necessitando suporte de NP domiciliar.

\section{Componentes da nutrição parenteral}

1. Fluidos

Os determinantes práticos que estabelecem as necessidades hídricas são o peso, a diurese e a densidade urinária. Débitos urinários entre 20 e $24 \mathrm{ml} / \mathrm{kg} / \mathrm{dia}$ e densidades urinárias entre 1004 e 1010 geralmente indicam oferta adequada de volume. A recomendação atual é bem ampla, situando as necessidades entre 60 e $180 \mathrm{ml} / \mathrm{kg} / \mathrm{dia}$, ressaltando-se que quanto menor e mais imaturo o RN, maiores serão suas perdas insensíveis e, consequentemente, suas necessidades hídricas ${ }^{35}$.

Quadro 1. Necessidades hídricas diárias, segundo o peso de nascimento $(\mathrm{PN})$ e a idade do recém-nascido

\begin{tabular}{|l|c|c|c|}
\hline $\mathbf{P N}(\mathbf{g})$ & $\mathbf{1}^{\mathbf{0}} \mathbf{\text { e 2}} \mathbf{2}^{\mathbf{~}} \mathbf{d i a}$ & $\mathbf{3}^{\mathbf{o}} \mathbf{\text { dia }}$ & $\mathbf{1 5}^{\mathbf{0}} \mathbf{- 3 0 ^ { \mathbf { 0 } }} \mathbf{\text { dia }}$ \\
\hline $750-1000$ & 105 & 140 & 150 \\
$1001-1250$ & 100 & 130 & 140 \\
$1251-1500$ & 90 & 120 & 130 \\
$1501-2000$ & 80 & 110 & 130 \\
32001 & 70 & 80 & 130 \\
\hline
\end{tabular}


Fototerapia: aumentar 20-30ml/kg/dia.

Calor radiante: aumentar 20-30 ml/ $\mathrm{kg} / \mathrm{dia}$.

Suporte ventilatório mecânico: diminuir $30 \%$.

\section{Energia}

As necessidades calóricas de um RN em crescimento, alimentado por via parenteral, variam de 80 a $90 \mathrm{kcal} / \mathrm{kg} / \mathrm{dia}$. Esses valores são basais, devendo ser ajustados de acordo com a atividade, temperatura corpórea, estresse e estados hipercatabólicos. Em algumas situações, atualmente bastante comuns, como na displasia broncopulmonar, as necessidades calóricas podem chegar a $150 \mathrm{kcal} / \mathrm{kg} / \mathrm{dia}$, o que não se obtêm exclusivamente com a $\mathrm{NP}^{23}$.

\section{Carboidratos}

O carboidrato universalmente utilizado em NP é a glicose, embora o RNPT apresente uma limitada capacidade em metabolizá-la. Nesses RN recomenda-se uma velocidade inicial de infusão de $4 \mathrm{mg} / \mathrm{kg} / \mathrm{min}$, pelo risco de apresentarem hiperglicemia e glicosúria, podendo ser elevada até $6 \mathrm{mg} / \mathrm{kg} / \mathrm{min}$. Incrementos acima deste valor devem ser feitos lentamente e com monitorização laboratorial. Nos RNT a velocidade inicial de infusão é de $6 \mathrm{mg} / \mathrm{kg} / \mathrm{min}$, podendo ser elevada até $10 \mathrm{mg} / \mathrm{kg} / \mathrm{min}$. A taxa de infusão de glicose depende fundamentalmente da tolerância particular de cada criança e o objetivo maior é promover aporte calórico adequado, sem provocar hiperglicemia em níveis potencialmente deletérios, como a hiperosmolaridade e a desidratação ${ }^{19}$.

\section{Proteínas}

As proteínas são indispensáveis para o crescimento, embora sejam de pouca valia como fonte energética. $\mathrm{O}$ nitrogênio deve ser fornecido em quantidade adequada à síntese protéica requerida para um crescimento normal e para a renovação e o reparo tecidual. Os RN em NP recebem nitrogênio através das soluções de aminoácidos (essenciais e não essenciais), com o objetivo de promover um aminograma plasmático semelhante ao perfil de um $\mathrm{RN}$ alimentado com leite humano ${ }^{36}$.

A tendência atual é de se introduzir a solução de aminoácidos já no primeiro dia da administração da NP, iniciando-se com $1 \mathrm{~g} / \mathrm{kg} / \mathrm{dia}$, aumentando-se $0,5 \mathrm{a} 1 \mathrm{~g} /$ $\mathrm{kg} / \mathrm{dia}$, até se atingir 3 a 3,5 g/ $/ \mathrm{kg} /$ dia. Para a máxima utilização do nitrogênio deve haver uma relação de 1/ 160 a 1/200 entre gramas de Nitrogênio e calorias não protéicas, lembrando que $6,25 \mathrm{~g}$ de aminoácidos fornecem 1 grama de Nitrogênio.

As soluções habitualmente prescritas são: Aminoped 10\%, Aminoplasmal 10\% e Pediamino PLM $10 \%$.
Quadro 2. Composição da solução de aminoácidos $\left(\right.$ Aminoped $\left.^{\mathrm{R}}\right) \mathbf{1 0 \%}$

\begin{tabular}{|c|}
\hline Aminoácidos essenciais \\
\hline L - isoleucina: - $6,4 \mathrm{~g} / 1$ \\
\hline $\mathrm{L}$ - leucina - $10,75 \mathrm{~g} / \mathrm{l}$ \\
\hline $\mathrm{L}$ - lisina $-7,09 \mathrm{~g} / 1$ \\
\hline $\mathrm{L}$ - metiona $4,62 \mathrm{~g} / \mathrm{l}$ \\
\hline $\mathrm{L}$ - fenilalanina $4,57 \mathrm{~g} / \mathrm{l}$ \\
\hline $\mathrm{L}-$ treonina $-5,15 \mathrm{~g} / \mathrm{l}$ \\
\hline $\mathrm{L}$ - triptofano - $1,83 \mathrm{~g} / \mathrm{l}$ \\
\hline $\mathrm{L}$ - valina - 7,09 g/1 \\
\hline L - cisteína - 0,38 g/l \\
\hline Aminoácidos semi-essenciais \\
\hline $\mathrm{L}$ - arginina $-6,40 \mathrm{~g} / \mathrm{l}$ \\
\hline $\mathrm{L}$ - histidina - $4,14 \mathrm{~g} / \mathrm{l}$ \\
\hline Aminoácidos não essenciais \\
\hline Glicina - 4,14 g/l \\
\hline $\mathrm{L}$ - alanina $-7,16 \mathrm{~g} / \mathrm{l}$ \\
\hline $\mathrm{L}$ - serina - 9,03 g/l \\
\hline $\mathrm{L}$ - prolina $-16,19 \mathrm{~g} / \mathrm{l}$ \\
\hline $\mathrm{L}$ - tironina $-5,49 \mathrm{~g} / 1$ \\
\hline
\end{tabular}

5. Gorduras

Os lípides são as fontes calóricas de maior densidade, fornecendo 9 calorias por grama. A grande vantagem da adição de gorduras na NP, principalmente no período neonatal, reside no alto valor calórico associado à isotonicidade, facilitando sua administração inclusive através de veias periféricas, além de, já em pequenas quantidades $(0,5 \mathrm{~g} / \mathrm{kg} / \mathrm{dia})$ prevenirem a deficiência de ácidos graxos. As soluções contendo óleo de soja são as mais recomendadas por apresentarem quantidades maiores de ácido linolênico ${ }^{23}$.

Atualmente recomendam-se as soluções a 20\%, pela melhor relação entre as gorduras e os fosfolípides e maior densidade calórica. Inicia-se com $0,5 \mathrm{~g} / \mathrm{kg} / \mathrm{dia}$, em torno de $4^{\circ}$ ou $5^{\circ}$ dia de vida, com incrementos diários de $0,5 \mathrm{~g} / \mathrm{kg} / \mathrm{dia}$, até o máximo de $3,5 \mathrm{~g} / \mathrm{kg} / \mathrm{dia}$. Nos RNPT, os níveis de triglicérides e colesterol devem ser verificados mais amiúde, pois essas crianças são menos tolerantes às gorduras. Níveis de triglicérides acima de $200 \mathrm{mg} / \mathrm{dl}$ justificam a diminuição ou a suspensão da gordura ofertada endovenosamente.

Os riscos potenciais do excesso de lípides incluem depósito de gordura no sistema reticuloendotelial, com consequente comprometimento da sua função, além de plaquetopenia e diminuição da capacidade de difusão do oxigênio em território pulmonar ${ }^{23}$.

A solução de gordura é habitualmente infundida em 24 horas, não excedendo a velocidade de $0,15 \mathrm{~g} / \mathrm{kg} / \mathrm{hora}$. Em vigência da fase aguda da sepse, plaquetopenia ou hiperbilirrubinemia deve-se diminuir a quantidade infundida de lípides.

As soluções habitualmente prescritas são: Intralipid 20\%, Lipofundim MTC 20\% e Lipovenus $20 \%$. 
Quadro 3. Composição da solução de gorduras (Intralipid ${ }^{\mathrm{R}}$ ) $20 \%$

Intralipid $^{\mathrm{R}}(20 \%)$ : óleo de soja - 200g

fosfolípide da gema de ovo - $12 \mathrm{~g}$

glicerol - 22,5g

água destilada qsp - $1000 \mathrm{ml}$

\section{Eletrólitos ${ }^{34}$}

As necessidades eletrolíticas variam com a condição clínica do RN, sendo dependente de vários fatores, como a função renal, o estado de hidratação e o uso de diuréticos.

\section{A. Sódio}

As necessidades básicas de sódio giram em torno de $3 \mathrm{mEq} / \mathrm{kg} / \mathrm{dia}$, porém os $\mathrm{RN}$ de muito baixo peso podem necessitar de até $8 \mathrm{mEq} / \mathrm{kg} / \mathrm{dia}$; nesta condição é imprescindível controle laboratorial frequente. Habitualmente o sódio é introduzido a partir de 48 horas de vida, sendo que 2 a $4 \mathrm{mEq} / \mathrm{kg} / \mathrm{dia}$, normalmente, são suficientes para manter níveis séricos em torno de 130 a $150 \mathrm{mEq} / \mathrm{l}$.

\section{B. Potássio}

As necessidades habituais de potássio estão em torno de 2 a $3 \mathrm{mEq} / \mathrm{kg} /$ dia, devendo a oferta ser iniciada a partir de 48 horas de vida, logo após a estabilização da diurese.

Nos RN de muito baixo peso, em virtude de uma menor taxa de filtração glomerular, aliada à presença de acidose metabólica e balanço calórico negativo, os níveis de potássio, nos primeiros dias de vida, podem ser mais elevados, devendo-se assim, evitar a reposição sem determinação sérica prévia.

\section{Cloro}

As necessidades diárias variam de 2 a $3 \mathrm{mEq} / \mathrm{kg} / \mathrm{dia}$, sendo fornecido em forma de $\mathrm{NaCl}$ e $\mathrm{KCl}$.

\section{Acetato}

$\mathrm{O}$ acetato, in vivo, é rapidamente metabolizado em bicarbonato e, portanto, pode ser utilizado para a prevenção da acidose metabólica. O íon acetato é compatível com todos os componentes da NP, não provocando precipitações. Ainda não foram estabelecidas as necessidades diárias de acetato.

\section{Minerais ${ }^{34}$}

\section{A. Cálcio}

As necessidades básicas de cálcio variam de 10 a $50 \mathrm{mg} / \mathrm{kg} /$ dia de cálcio elementar. Essas quantidades devem ser fornecidas sob a forma de gluconato e glicerofosfato de cálcio, porém os sais de cálcio e os fosfatos formam complexos insolúveis nas soluções, quando em concentrações elevadas e, nem sempre é possível fornecer as quantidades ideais de cálcio e fósforo que preveniriam a doença metabólica óssea.

\section{B. Fósforo}

Além da função estrutural, o fósforo exerce ação no transporte de energia e liberação de oxigênio, influenciando ainda a fagocitose leucocitária. As recomendações habituais de fósforo variam de 0,5 a 2,0 mMol/kg/dia (20 a $60 \mathrm{mg} / \mathrm{kg} / \mathrm{dia})$.

Relação Cálcio/Fósforo:

No leite humano esta relação é de 2, permitindo assim, uma mineralização óssea adequada. A relação das soluções utilizadas na NP situam-se em torno de 1,7, relativamente próxima da situação ideal. Relações inversas podem causar hipocalcemia, aumento na secreção do paratormônio e osteopenia.

\section{Magnésio}

As doses habituais de manutenção situam-se entre 0,6 e 1,0 mEq/kg/dia (7 e $10 \mathrm{mg} / \mathrm{kg} / \mathrm{dia}$ ). Recomenda-se a monitorização sérica antes da reposição de doses mais elevadas de magnésio.

Quadro 4. Soluções utilizadas na NP e seus equivalentes por $\mathrm{ml}$

\begin{tabular}{|l|l|l|}
\hline Sódio & $\begin{array}{l}\text { NaCl 20\% } \\
\text { Acetato de sódio 10\% } \\
\text { Acetato de sódio 27,2\% }\end{array}$ & $\begin{array}{l}1 \mathrm{ml}=3,4 \mathrm{mEq} \\
1 \mathrm{ml}=2,0 \mathrm{mEq} \\
1 \mathrm{ml}=0,74 \mathrm{mEq}\end{array}$ \\
\hline Potássio & $\mathrm{KCl} 19,1 \%$ & $1 \mathrm{ml}+2,5 \mathrm{mEq}$ \\
& $\mathrm{KCl} 2 \mathrm{mEq} / \mathrm{ml}$ & $1 \mathrm{ml}=2,0 \mathrm{mEq}$ \\
\hline Cálcio & Gluconato de cálcio 10\% & $1 \mathrm{ml}=1,5 \mathrm{mEq}$ \\
\hline \multirow{2}{*}{ Fósforo } & Fosfato diácido de $\mathrm{K} \mathrm{25 \%}$ & $1 \mathrm{ml}=1,8 \mathrm{mEq}$ \\
\hline \multirow{2}{*}{ Magnésio } & $\begin{array}{l}\text { Fosfato monoácido de } \mathrm{K} 25 \% \\
410 \%\end{array}$ & $\begin{array}{l}1 \mathrm{ml}=2,9 \mathrm{mEq} \\
1 \mathrm{ml}=0,8 \mathrm{mEq} \\
1 \mathrm{ml}=1,0 \mathrm{mEq} \\
1 \mathrm{ml}=4,0 \mathrm{mEq}\end{array}$ \\
\hline
\end{tabular}

8. Vitaminas ${ }^{21}$

As vitaminas são fornecidas em soluções multivitamínicas, que são adicionadas à solução parenteral. As doses enterais recomendadas servem apenas como base, já que as soluções endovenosas podem sofre influências em relação ao recipiente utilizado e à fotodegradação.

As vitaminas hidrossolúveis compreendem a vitamina $\mathrm{C}$ e as do complexo $\mathrm{B}$. As quantidades dessas vitaminas, por via endovenosa, devem ser maiores do que as ofertadas por via enteral, pois uma grande parte é excretada pelos rins. Elas são habitualmente adicionadas à NP em quantidades três vezes maiores do que as doses orais.

As vitaminas lipossolúveis compreendem as vitaminas A, D, E e K. Essas vitaminas, quando ofertadas em excesso, podem acumular-se no organismo, com risco de provocarem intoxicações.

As soluções vitamínicas não contêm a vitamina $\mathrm{K}$. Esta deve ser ofertada, semanalmente, na dose de $1 \mathrm{mg}$, por via intramuscular. 
Quadro 5. Composição das soluções multivitamínicas (Polivit $A^{\mathrm{R}}$ e Polivit $\mathbf{B}^{\mathrm{R}}$ )

\begin{tabular}{|c|c|c|c|}
\hline Polivit $\mathrm{A}^{\mathrm{R}}$ : & $\begin{array}{l}\text { Vitamina A - } 2300 \mathrm{Ui} \\
(10 \mathrm{ml}) \text { Vitamina D - } 400 \mathrm{Ui} \\
\text { Vitamina E - } 7 \mathrm{mg} \\
\text { Vitamina B }-1,2 \mathrm{mg} \\
\text { Vitamina B }-17 \mathrm{mg} \\
\text { Vitamina }{ }^{3}-5 \mathrm{mg} \\
\text { Vitamina } \text { B }^{5}-1 \mathrm{mg} \\
\text { Vitamina } C^{6}-80 \mathrm{mg}\end{array}$ & $\begin{array}{l}\text { Polivit } \mathrm{B}^{\mathrm{R}} \text { : } \\
(5 \mathrm{ml})\end{array}$ & $\begin{array}{l}\text { Vitamina } B_{7}-20 m g \\
\text { Vitamina } B^{7}-140 \mathrm{mg} \\
\text { Vitamina } B^{9}-1 \mathrm{mg} \\
\text { Vitamina } B_{2}^{12}-1,4 \mathrm{mg}\end{array}$ \\
\hline
\end{tabular}

Polivit A = $4 \mathrm{ml} / \mathrm{dia}$ (RNMBP) e 6,5 ml/dia (RNT e RNPT > $1500 \mathrm{~g}$ ) Polivit $\mathrm{B}=2 \mathrm{ml} / \mathrm{dia}$ (RNT e RNPT)

\section{Oligoelementos ${ }^{34}$}

Apesar das soluções básicas de NP não conterem oligoelementos, estes podem estar presentes como contaminantes, além da sua presença no sangue e outros hemoderivados que essas crianças possam receber.

Os sinais clínicos da deficiência de oligoelementos dificilmente aparecem antes da $4^{\text {a }}$ semana de vida e, a possibilidade de se ofertar dieta enteral, mesmo em pequenas quantidades, já são suficientes para prevenir a deficiência.

Quadro 6. Necessidades de oligoelementos e composição de Oliped ${ }^{\circledR}$ e Neo-zinc ${ }^{\circledR}$

\begin{tabular}{|l|cc|l|l|}
\hline Oligoelemento & \multicolumn{2}{|c|}{$\begin{array}{c}\text { Necessidade diária } \\
(\mathrm{mcg} / \mathrm{kg} / \text { dia })\end{array}$} & $\begin{array}{l}\text { Neo-zinc® } \\
(\mathrm{mcg} / \mathrm{ml})\end{array}$ & $\begin{array}{l}\text { Oliped® } \\
(\mathrm{mcg} / \mathrm{ml})\end{array}$ \\
\hline Termo & Pré-termo & & 200 & 100 \\
Cobre (mcg) & 20 & 20 & & 20 \\
Manganês (mcg) & 1 & 1 & & 6 \\
Cromo (mcg) & 0,2 & 0,2 & & 0,17 \\
\hline
\end{tabular}

RNT - Oliped $(1 \mathrm{ml} / \mathrm{kg} / \mathrm{d})+$ Neo-zinc $(0,75 \mathrm{ml} / \mathrm{kg} / \mathrm{d})$;

RNPT - Oliped $(1 \mathrm{ml} / \mathrm{kg} / \mathrm{d})+$ Neo-zinc $(1,5 \mathrm{ml} / \mathrm{kg} / \mathrm{d})$

\section{Controles clínicos e laboratoriais}

Nos RN submetidos à NP os seguintes parâmetros devem ser avaliados:

- Peso: diário;

- Perímetro cefálico: semanal;

- Comprimento: semanal;

- Glicemia: no mínimo 1 vez por dia;

- Eletrólitos: 1 vez por semana;

- Uréia e creatinina: 1 vez por semana;

- Transaminases: 1 vez cada 2 semanas;

- Proteínas (total e frações): 1 vez cada 2 semanas;

- Hematócrito e hemoglobina: 1 vez por semana e;

- Triglicérides: 1 vez por semana.

Em RNPT, principalmente menores do que 32 semanas ou $1500 \mathrm{~g}$, deve-se determinar cálcio, fósforo e fosfatase alcalina na $2^{\mathrm{a}}$ semana de vida e depois, a cada 15 dias.

O ideal é que todos essas determinações laboratoriais sejam realizadas através de micrométodos, para se evitar a espoliação de sangue e a necessidade de transfusões.

\section{Complicações da nutrição parenteral}

1 - Relativas à técnica de infusão:

- mau posicionamento do catéter;

- tromboses;

- tromboflebites;

- infiltrações;

- infecções.

2 - Relativas à limitada capacidade metabólica do recém-nascido:

- hiperglicemia pela oferta excessiva, ou por alterações em seu estado basal (infecções), ou por irregularidades no fluxo de infusão;

- hipoglicemia por interrupção da infusão, por uso inadequado de insulina ou na presença de infecções;

- hiperazotemia pela oferta excessiva de nitrogênio;

- alterações eletrolíticas, de minerais e de vitaminas, por oferta inadequada;

- hipertrigliceredemia e aumento de ácidos graxos, por oferta lipídica aquém da capacidade do recém-nascido.

3 - Relativas à própria infusão:

- aminograma alterado;

- hipercolesterolemia;

- hiperfosfolipidemia.

4 - Relativas ao método:

- hipotrofia da mucosa digestiva;

- alterações hepáticas. 
Falcão MC. Nutrition support in the ill or premature newborn infant. Rev Med (São Paulo) 2003 jan.dez:;82(1-4):11-21.

\begin{abstract}
The newborns need to adapt from a relative constant supply of all nutrients to an intermittent feeding. Normal term infants rapidly overcome this situation. However, preterm newborns or even ill term infants commonly exhibit special condition that injure their nutrition. In these cases, principles of nutritional support should be establish, in order to improve nutritional goals. The principles of nutritional support are: growth patterns, fetal body composition, nutritional assessment including growth and metabolic parameters, nutrient requirements and nutritional monitorization.
\end{abstract}

KEYWORDS: Nutritional support. Nutritional assessment. Infant, newborn. Infant, premature. Nutritional requirements. Infant nutritional.

\title{
REFERÊNCIAS
}

1. Gluckman PD. Endocrine and nutritional regulation of prenatal growth. Acta Paediatr Suppl. 1997;423:153-7.

2. Brooke OG, Wood C, Butters F. The body proportions for small-for-dates infants. Early Hum Dev 1984;10:85-94.

3. Ramos JLA. Avaliação do crescimento intra-uterino por medidas antropométricas do recém-nascido [tese]. São Paulo: Faculdade de Medicina, Universidade de São Paulo; 1983.

4. Falcão MC. Avaliação nutricional e metabólica do recémnascido. In: Falcão MC, Carrazza FR, editores. Manual básico de apoio nutricional em pediatria. São Paulo: Atheneu; 1999, p.11-5.

5. Falcão MC. Avaliação nutricional do recém-nascido. Rev Paul Pediatr 1997;15:219-20.

6. Hadlock FP, Deler RL, Harrist RB, Park SK. Estimated age: computer assisted analysis of multiple fetal growth parameters. Radiology 1984,152:497-501.

7. Capurro H, Konichesky W, Fonseca D, Caldeyro-Barcia R. A simplified method for diagnosis of gestacional age in the newborn infant. J Pediatr 1978,93:120-2.

8. Dubowitz IM, Dubowitz V, Goldberg C. Clinical assesment of gestacional age in the newborn infant. J Pediatr 1970,77:1-10.

9. Ballard JL, Khoury JC, Wedig K, Wang L, Eilers-Walsman BL, Lipp R. New Ballard score expanded to include extremely premature infants. J Pediatr 1991;119:417-23.

10. Battaglia FC, Lubchenko LO. A practical classification of newborn infants by weight and gestational age. J Pediatr 1967,71:159-63.

11. Kramer MS, Oliver M, Mclean FH, Wilis DM, Usher RH. Impact of intrauterine growth retardation and body proportionatity on fetal and neonatal outcome. Pediatrics 1990;84:717-23.

12. Georgieff MK, Sasanow SR. Nutritional assessment of the neonate. Clin Perinatol 1986;13:73-89.

13. Sasanow SR, Georgieff MR, Pereira GR. Mid-arm circumference and mid-arm circumference/head ratios; standard curves for anthropometric assessment of neonatal nutritional status. J Pediatr 1986;109:311-5.

14. Thornton CJ, Shannon DL, Hunter MA, Brans YW. Dynamic skinfold thickness measurements: a noninvasive estimate of neonatal extracellular water content. Pediatr Res 1982;16:989-94.
15. Wolfe HM, Brans YW, Gross TL, Bhatia KK, Sokol RJ. Correlation of commonly used measures of intrauterine growth with estimated neonatal fat. Biol Neonate 1990;57:167-71.

16. Costarino Jr AT, Baumgart S. Water as nutrition. In: Tsang RC, Lucas A, Uauy R, Zlotkin S, editors. Nutritional needs of the preterm infant. Baltimore: Williams and Wilkins; 1993. p.1-14.

17. Costarino Jr AT, Baumgart S. Modern fluid and electrolyte management of the critically ill premature infant. Pediatr Clin North Am 1986;33:153-78.

18. Putet G. Energy. In: Tsang RC, Lucas A, Uauy R, Zlotkin S, editors. Nutritional needs of the preterm infant. Baltimore: Willians and Wilkins; 1993. p.15-28.

19. American Academy of Pediatrics. Committee on Nutrition. Nutritional needs of low-birth-weight infants. Pediatrics 1985;75:976-86.

20. Sun Y, Awnetwart EL, Collier SB, Galagher LM, Olsen JE, Stewart JE. Nutrition. In: Cloherty JP, Stark AR, editors. Manual of neonatal care. 4th ed. Philadelphia: Little Brown; 1998. p.101-34.

21. Greene HL. Vitamins for newborn infants formulas. Eur J Clin Nutr 1991;46:1-8.

22. Pereira GR, Georgieff MK. Nutritional assessment. In: Polin RA, Fox WW, editors. Fetal and neonatal physiology. Philadelphia: WB Saunders; 1992. p.277-85.

23. Pereira GR. Nutritional care of the extremely premature infant. Clin Perinatol 1995;22:61-75.

24. Atkinson AS, Radek IC, Anderson GH. Macromineral balances in premature infants fed their own mothers'milk or formula. J Pediatr 1983;102:99-106.

25. Atkinson AS. Human Milk feeding of the micropremie. Clin Perinatol 2000;27:235-47.

26. European Society of Paediatric Gastroenterology and Nutrition (ESPGAN). Committee on Nutrition. Guidelines on infant nutrition. I. Recomendations for the compositrion of an adapted formula. Acta Paediatr Scand Suppl. 1977; 262(suppl):1-20.

27. Schanler RJ. The role of human milk fortification for premature infants. Clin Perinatol 1998;25:645-57. 


\section{Falcão MC. Suporte nutricional no récem-nascido.}

28. Schanler RJ, Garza C. Improved mineral balance in very low birt weight fed firtified human milk. J Pediatr 1987;112:452-6.

29. Schanler RJ, Shulman RJ, Lau C. Feeding strategies for premature infants: beneficial outcomes of feeding fortified human milk versus preterm formula. Pediatrics 1999;103:1150-7.

30. Kashyap S, Schulze KF, Forsyth M, Dell RB, Ramakrishnan R, Heird WC. Growth, nutrient retention and metabolic response of low-birth-weight infants fed supplemented and unsupplemented preterm human milk. Am J Clin Nutr 1990;52:254-62.

31. Wojnar MM, Hawkins WG, Lang CH. Nutritional support of the septic patient. Critical Care Clin 1995;11:717-33.

32. Chwals WJ. Overfeeding the critically ill child: fact or fantasy.
New Horizon 1994;2:147-55.

33. Adan D, La Gamma, EF, Browne LE. Nutritional management and the multisystem organ failure/systemic inflammatory response syndrome in critically ill preterm neonates. Critical Care Clin 1995;11:751-84.

34. Heird WC, Gomez MR. Parenteral nutrition. In: Tsang, RC, Lucas A, Uauy R, Zlotkin S, editors. Nutritional needs of the preterm infant. Baltimore: Willians and Wilkins; 1993. p.225-42.

35. Nash MA. The management of fluid and electrolyte disorders in the neonate. Clin Perinatol 1981;8:251-62.

36. Melnik G. Value of specialty intravenous amino acid solutions. Am J Health Syst Pharm 1996;53:671-4. 